\title{
Cognitive-behavioural therapy effects on employment- related outcomes for individuals with mental illness: A systematic review
}

\begin{tabular}{|c|c|}
\hline $\begin{array}{l}\text { Authors: } \\
\text { Kim Minjoo }^{1} \\
\text { Elias Mpofu }^{1} \\
\text { Kaye Brock } \\
\text { Michael Millir } \\
\text { James Athana }\end{array}$ & $\begin{array}{l}\text { gton }^{1} \\
\text { sou }^{1}\end{array}$ \\
\hline \multicolumn{2}{|c|}{$\begin{array}{l}\text { Affiliations: } \\
{ }^{1} \text { Discipline of Rehabilitation } \\
\text { Counselling, University of } \\
\text { Sydney, Australia }\end{array}$} \\
\hline \multicolumn{2}{|c|}{$\begin{array}{l}\text { Correspondence to: } \\
\text { Elias Mpofu }\end{array}$} \\
\hline \multicolumn{2}{|c|}{$\begin{array}{l}\text { Email: } \\
\text { elias.mpofu@sydney.edu.au }\end{array}$} \\
\hline \multicolumn{2}{|c|}{$\begin{array}{l}\text { PO Box } 170 \text {, Lidcombe, New } \\
\text { South Wales } 1825 \text {, Australia }\end{array}$} \\
\hline \multicolumn{2}{|c|}{$\begin{array}{l}\text { Received: } 10 \text { Dec. } 2013 \\
\text { Accepted: } 13 \text { Mar. } 2014 \\
\text { Published: } 06 \text { June } 2014\end{array}$} \\
\hline \multicolumn{2}{|c|}{$\begin{array}{l}\text { How to cite this article: } \\
\text { Minjoo, K., Mpofu, E., } \\
\text { Brock, K., Millington, M. } \\
\text { \& Athanasou J. (2014). } \\
\text { Cognitive-behavioural } \\
\text { therapy effects on } \\
\text { employment-related } \\
\text { outcomes for individuals } \\
\text { with mental illness: A } \\
\text { scoping ystematic review. } \\
\text { SA Journal of Industrial } \\
\text { Psychology/SA Tydskrif vir } \\
\text { Bedryfsielkunde, 40(2), Art. } \\
\text { \#1188, } 6 \text { pages. http:// } \\
\text { dx.doi.org/10.4102/sajip. } \\
\text { v40i2.1188 }\end{array}$} \\
\hline \multicolumn{2}{|c|}{$\begin{array}{l}\text { Copyright: } \\
\text { (C) 2014. The Authors. } \\
\text { Licensee: AOSIS } \\
\text { OpenJournals. This w } \\
\text { is licensed under the } \\
\text { Creative Commons } \\
\text { Attribution License. }\end{array}$} \\
\hline \multicolumn{2}{|l|}{ Read online: } \\
\hline 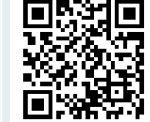 & $\begin{array}{l}\text { code with your } \\
\text { smart phone or } \\
\text { mobile device } \\
\text { to read online. }\end{array}$ \\
\hline
\end{tabular}

Orientation: To identify the effects of interventions in cognitive-behaviour therapy (CBT) on employment-related outcomes world-wide for individuals with mental illness.

Research purpose: A search of the relevant literature was conducted through PsychInfo, Medline, Scopus and Google Scholar ${ }^{\mathrm{TM}}$, covering the period between 1995 and August 2011. The methodological quality of included studies was assessed using the criteria from Evidence Based Library and Information Practice (EBLIP). Eleven studies met the inclusion criteria.

Motivation for the study: Evidence is needed on best practices to support work participation by people with mental illness. Effective cognitive-behavioural intervention might enhance their personal control over participation in employment aside from systemic or policy-oriented interventions.

Research approach, design and method: A scoping review was done to map trends in the evidence for CBT as an intervention to support employment participation by people with mental illness. A scoping review is exploratory, the evidence of which lays the basis for subsequent studies. The methodological quality of included studies was assessed using the EBLIP Critical Appraisal Checklist.

Main findings: Cognitive-behaviour therapy was an effective intervention approach for better work productivity, longer work hours, higher re-employment rate and enhanced mental health for individuals with mental illness.

Practical/managerial implications: Cognitive-behaviour therapy is a promising strategy for industrial and organisational psychologists dealing with people who have a mental illness. It enhances employment and maintains work adjustment. Additional clinical trials in diverse populations and contexts will further establish its efficacy.

Contribution/value-add: This scoping review aggregated the preliminary evidence for the efficacy of cognitive-behaviour therapy as a work-participation intervention for people with mental illness.

\section{Introduction}

Mental illness is a term that varies in its breadth and depth but is associated with an emotional or behavioural disorder (American Psychiatric Association, 2013). Approximately one in four people in the general population are affected by mental illness at some point in their lives (Kessler, Merikangas \& Wang, 2008; World Health Organization [WHO], 2001). Naturally, mental illness is associated with functional impairments that interrupt life activities (New Freedom Commission on Mental Health, 2003). Specifically, severe mental illness often impairs one's capacity to choose, obtain and keep a job and to earn a living (Tsang, Lam, Ng \& Leung, 2000). Furthermore, WHO (2007) viewed the complementary condition of mental health as a state of well-being that allows someone to work. It is recognised that there are critical barriers to employment for people with mental illness (Lysaker, Davis, Bryson \& Bell, 2009). These include difficulties with interpersonal relationships and with the completion of tasks at work. There are concomitant issues in coping with stigma and work stress as well as low levels of self-efficacy or self-defeating beliefs (see also Marwaha \& Johnson, 2004).

Despite these issues, employment has been considered a key component of recovery from mental illness (see Link, Yang, Phelan \& Collins, 2004). It is an essential aspect because it is linked with social, economic and personal advancement (Garske \& Stewart, 1999). For people with mental illness, meaningful vocational activities can help develop valued societal roles, reduce stigmatisation, increase social connectedness, serve as a normalising factor and enhance quality of life (Marwaha 
\& Johnson, 2004). In contrast, chronic unemployment is linked with isolation, poverty and a diminishing self-worth that hinder efforts at recovery (Mak, Tsang \& Cheung, 2006). Nevertheless, although people with mental illness are eager to work and can be employed successfully, the participation rate of persons with mental disorders was only around 38\% in 1990 and continued at this historical low with major schooling and employment restrictions (Australian Bureau of Statistics, 1990:26; Australian Bureau of Statistics, 2013). To date, employment remains a most challenging aspect of recovery for people with mental illness.

Unemployment not only hinders recovery at the individual level but also has an obvious cost to society in the form of the loss of human-resource potential. This is accompanied by massive medical and social-security costs. For instance, in Australia, there are 3.1 million people with a mental illness, and the estimated medical cost alone is some \$A2.7 billion (Australian Bureau of Statistics, 2008). In comparison to the direct cost for care of people with mental illness (e.g. medication, clinic visits, hospitalisation), nearly twothirds of the overall loss is indirect costs such as reduced labour supply, public income-support payments, reduced educational attainment, incarceration, homelessness or medical complications associated with mental illness. Insel (2012) reported the estimated overall economic burden of mental illness in the United States of America as \$US317 billion and the loss of earnings for people with mental illness at approximately \$US193.2 billion. In summary, there are positive consequences to enhancing recovery from mental illness through treatment interventions.

Cognitive-behaviour therapy is one intervention that has been applied to people with emotional psychological and psychiatric difficulties. Rachman (1997) traced its history from a combination of behaviour-modification approaches with cognitive therapies to a short term, focused approach to dealing with a specific problem. This approach centres on changing the thoughts and feelings that influence behaviour. The emphasis is on learning new skills or habits in areas such as mindfulness or acceptance and commitment. The essential component is the formation of new patterns of thinking. Its effectiveness has been confirmed in a number of studies (e.g. Butler, Chapman, Forman \& Beck, 2006).

In particular, cognitive-behaviour therapy has been applied to address impairments of vocational functioning amongst people with mental illness. Approaches to cognitivebehaviour therapy are positively associated with employment outcomes for people with mental illness. These include expectancy for employment success (Rose \& Perz, 2005), satisfaction with work supervision (Kidd, Boyd, Bieling, Pike \& Kazarian, 2008) and increased working hours or improved work status (Lysaker, Davis, Bryson \& Bell, 2009).

Cognitive-behaviour therapy incorporates diverse approaches that may focus on general improvements in cognitive functioning and social skills, managing negative and positive symptoms, reducing internalised stigma and enhancing self-efficacy or positive beliefs (see a review in Butler, Chapman, Forman \& Beck, 2006). It is not clear, however, what type of cognitive-behaviour therapy is best associated with specific employment outcomes. The objective of this article was to systematically summarise and synthesise the empirical evidence across studies concerning the effects of cognitive-behaviour therapy on employment outcomes for people with mental illness. The results of this study can provide a better understanding of the overall general or specific effects of cognitive-behaviour therapy on employment outcomes so that industrial psychologists can design or implement intervention programs.

\section{Literature search}

The process for the literature search is summarised in Figure 1. We located published articles in the English language dated between 1995 and 2011, investigating the effects of cognitive-behaviour therapy on vocational outcomes of people with mental illness. Searches were conducted using computerised databases, including PsychInfo, Medline, Scopus and Google Scholar ${ }^{\mathrm{TM}}$. In addition to the searches utilising online databases, manual searches were performed in the reference lists of the articles and electronic journals reviewed. The search was conducted using 19 keywords related to mental illness, cognitive-behaviour therapy and employment outcomes. The keywords were grouped under three principal headings: mental illness, cognitive-behaviour therapy and employment (a copy of the 19 specific terms used for the searches is available from the authors upon request).

The literature search resulted in a total of 445 references through the databases and manual searches. Of these, $86 \%$ were eliminated by reviewing the title, text or abstract on the grounds that they were not found to be sufficiently empirical, or they did not report any component of mental illness, cognitive-behaviour therapy or employment outcomes. The

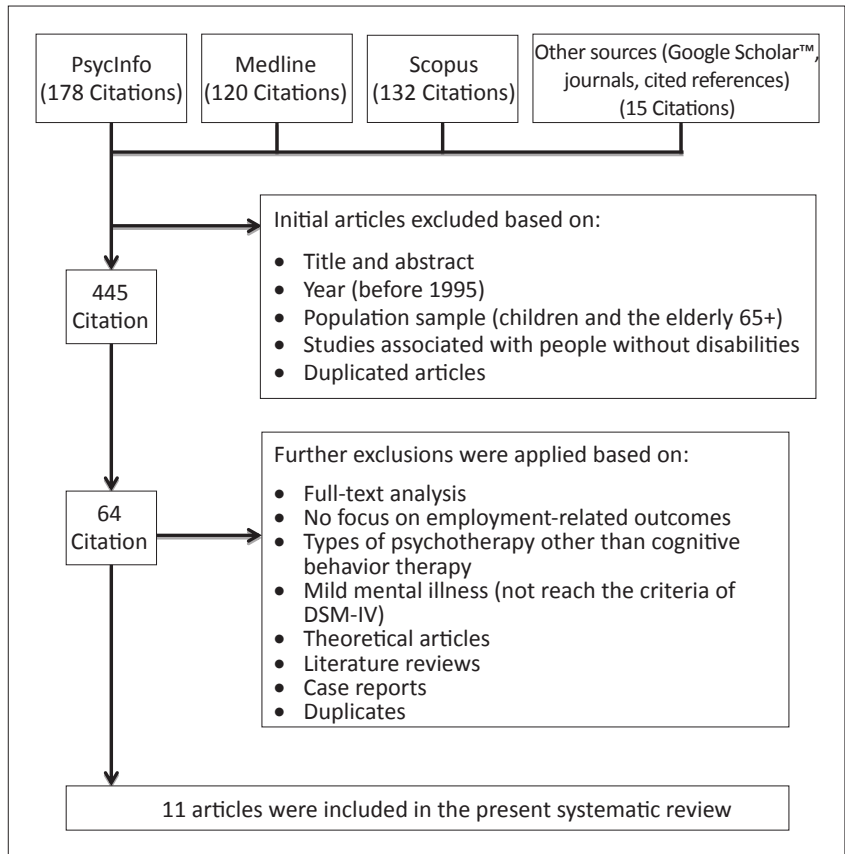

FIGURE 1: Summary of the study-selection process. 
remaining 64 articles were reviewed in detail, and of these, only 11 articles met the criteria listed (see Figure 1).

\section{Inclusion criteria}

The criteria for the inclusion of a study in this systematic review were the following: (1) the target population of the study was individuals of working age (18-65 years old) with mental illness; (2) cognitive-behaviour therapy was the intervention (independent variable) and it included descriptions of the specific approaches used during the study; (3) employmentrelated outcomes were the dependent variables, including employment rate, job satisfaction, employment productivity and working hours. Articles were excluded if they aimed at investigating the efficacy of cognitive-behaviour therapy interventions in general. Non-empirical studies such as case studies, review articles and book chapters were excluded. Dissertations were also not included in the study.

To assess the quality of included studies, the widely used Evidence Based Library and Information Practice critical appraisal (Glynn, 2006) were used. These tools evaluate a study across four areas: population, data collection, design and results. All studies scored more than $85 \%$ on the critical appraisal checklist, indicating good quality.

\section{Data extraction}

The following data were extracted from each article in order to examine the effects of cognitive behaviour therapy on employment outcomes: citation, study design, demographic information, sample size, mental illness diagnosis, intervention and measurements concerning cognitivebehaviour therapy, vocational-outcome measurement and results related to vocational outcome. Based on the description of the intervention approaches, cognitivebehaviour therapy was coded into three types of approaches: (1) general cognitive-behaviour therapy, (2) vocationallyoriented cognitive-behaviour therapy and (3) vocationallyoriented cognitive-behaviour therapy combined with employment services. Vocational outcomes were coded into vocational functioning, job satisfaction, employment status (employment rates or working hours), work adjustment and expectation of job success. In addition, outcome variables related to symptom alleviation, psychological functioning, psychosocial functioning, self-esteem and cognitive functioning were also included (see Table 1).

\section{Studies reviewed}

Detailed descriptions of the 11 studies included in the review are listed in Table 1. Amongst these 11 articles, six were conducted in the United States of America, two in the United Kingdom and one each in Canada, Switzerland and Australia. The total population size of the included articles is 623 and the number of participants in each study ranged from $19-139(\mathrm{M}=57)$. The mean age of participants was 33.7 years. In eight studies, there were more male participants than females. One study included only women participants (Washington, 1999), and two studies included only men (Lysaker et al., 2005; Lysaker, Davis \& Beatties, 2006). The types of mental-illness conditions included were the following: schizophrenia, depression, bipolar disorder, mood and anxiety disorders and substance dependence. Of the selected 11 studies, six were randomised controlled trials, two used a pre-test post-test design, two used quasiexperimental design, and one was a naturalistic trial.

In reviewing the descriptions of cognitive-behaviour therapy, five studies applied vocationally-oriented cognitivebehaviour therapy, four studies used general cognitivebehaviour therapy, and two used general cognitivebehaviour therapy in combination with vocationally oriented services as the interventions. Most studies used existing cognitive-behaviour therapy approaches or developed manuals to assure the standardisation of the intervention. Of these 11 studies, five studies applied group cognitivebehaviour therapy only, three provided group and individual cognitive-behaviour therapy, two provided individual cognitive-behaviour therapy, and one did not specify the type of intervention intervention.

\section{Findings}

Table 2 provides an overview of the number of statistically significant effects for each outcome variable and also for each type of cognitive behaviour therapy. Most studies examined more than one of the six vocational variables (vocational functioning, job satisfaction, employment status, work adjustment, work involvement, expectation of success, cognitive functioning) or more than one of the four variables related to mental illness (psychological functioning, psychosocial functioning, symptom, self-esteem). There were a total of 51 results from the 11 studies, and Table 2 provides a cross-categorisation. Each outcome variable is a row, and each type of treatment condition is a column. These are grouped into vocational-related outcomes ( $N=28$ results) or mental-illness related outcomes $(N=23$ results).

Of these 28 sets of vocational-related results, 16 (57.2\%) reported that participation in or the completion of a cognitivebehaviour therapy program significantly improved some aspect of vocational functioning. Statistically significant vocational outcomes were reported in 16 out of 28 results or just over half of all the outcomes. Four out of six studies improved employment status (this is the result shown at the end of the third row of the table). Five out of five studies showed improved employment. Work adjustment showed no statistically significant improvement. Three out of three studies showed a statistically significant improvement in work involvement. Expectation of success and cognitive functioning showed improvement in 0.5 and 0.6 out of 2 and 5 results, respectively. Looking at the row totals in the 10th row of Table 2, it appears that general cognitive-behaviour therapy plus vocational rehabilitation had the largest proportion of statistically significant workplace outcomes.

The effects of cognitive-behavioural interventions on vocational outcomes (0.57) were only marginally better than 
TABLE 1: Summary of studies included in this review.

\begin{tabular}{|c|c|c|c|c|c|}
\hline Study \& Lead & Study design & Participants & Types of interventions & $\begin{array}{l}\text { Types of outcomes and } \\
\text { measures }\end{array}$ & $\begin{array}{l}\text { Results }(p<.05 \text { is considered as } \\
\text { significant) }\end{array}$ \\
\hline $\begin{array}{l}\text { Bee } \\
(2010) \text { UK }\end{array}$ & $\begin{array}{l}\text { RCT } \\
2 \text { groups: } \\
\text { A: Telephone-cognitive } \\
\text { behaviour therapy }+ \\
\text { usual care }(n=26) \\
\text { B: usual care }(n=27)\end{array}$ & $\begin{array}{l}N=53 \\
\text { Mean age } 45 \\
\text { Mild / moderate mental } \\
\text { disorders }\end{array}$ & $\begin{array}{l}\text { General cognitive behaviour } \\
\text { therapy } \\
\text { A: } 12 \text { weeks general telephone } \\
\text { cognitive behaviour therapy } \\
\text { B: Usual care }\end{array}$ & $\begin{array}{l}\text { - Symptom } \\
\text { - Psychological functioning } \\
\text { - Work adjustment } \\
\text { - Employment status }\end{array}$ & $\begin{array}{l}\text { CORE-OM: n.s. } \\
\text { HADS: n.s. } \\
\text { WSAS: } \text {.s. } \\
\text { - Working hours: } d=17.2 \text {; e.s. } \\
=.75 ; p<.005\end{array}$ \\
\hline $\begin{array}{l}\text { Lysaker } \\
\text { (2009) USA }\end{array}$ & $\begin{array}{l}\text { RCT } \\
2 \text { groups: } \\
\text { A: IVIP }+26 \text {-week job } \\
\text { placement }(n=50) \\
\text { B: } 26 \text {-week job } \\
\text { placement }(n=50)\end{array}$ & $\begin{array}{l}N=100 \\
\text { Mean age: } 45.94 \\
\text { Male: } 85 \\
\text { Female: } 15 \\
\text { Schizophrenia or } \\
\text { schizoaffective disorder }\end{array}$ & $\begin{array}{l}\text { Vocationally-focused cognitive } \\
\text { behaviour therapy } \\
\text { A: } 8 \text { week of Weekly group and } \\
\text { individual cognitive behaviour } \\
\text { therapy sessions focusing. } \\
\text { B: Support services including } \\
\text { job placement. }\end{array}$ & $\begin{array}{l}\text { - Symptom } \\
\text { - Vocational functioning } \\
\text { - Employment status }\end{array}$ & $\begin{array}{l}\text { - PANSS: n.s. } \\
\text { - WBI: } t=2.05 ; p<.05 \text {; } \\
\text { - Working hours: } t=2.98 ; p<.01\end{array}$ \\
\hline $\begin{array}{l}\text { Vauth (2005) } \\
\text { Switzerland }\end{array}$ & $\begin{array}{l}\text { RCT } \\
\text { 3 groups: } \\
\text { A: CAST + vocational } \\
\text { rehabilitation (VR) }(n=47) \\
\text { B: TSSN + VR, }(n=45) \\
\text { C: only VR }(n=46)\end{array}$ & $\begin{array}{l}N=138 \\
\text { Mean age: } 28.8 \\
\text { Schizophrenia }\end{array}$ & $\begin{array}{l}\text { General cognitive behaviour } \\
\text { therapy } \\
8 \text { weeks intervention. A: } \\
\text { Focusing on general skills of } \\
\text { cognitive behaviour therapy } \\
\text { and deficits in attention, verbal } \\
\text { memory and planning. } \\
\text { B: TSSN focusing on improve } \\
\text { negative symptoms and } \\
\text { motivation + VR }\end{array}$ & $\begin{array}{l}\text { - Symptom } \\
\text { - Psychological functioning } \\
\text { - Cognitive functioning } \\
\text { - Employment status }\end{array}$ & $\begin{array}{l}\text { - PANSS: n.s. } \\
\text { - CDSS: } \mathrm{n} . \mathrm{s} \text {. } \\
\text { - Attention: n.s.; immediate } \\
\text { recall: } F(2,97)=3.8 ; p<.01 \text {; } \\
\text { delayed recall: } F(2,97)=3.6 \text {; } \\
p<.05 ; \text { Planning: n.s. } \\
\text { - Competitive work } 12 \\
\text { months follow up: CAST+VR } \\
\text { compared to VRA: chi-square } \\
=3.2 ; p<.05\end{array}$ \\
\hline $\begin{array}{l}\text { Lysaker } \\
\text { (2005) USA }\end{array}$ & $\begin{array}{l}\text { RCT } \\
2 \text { groups: } \\
\text { A: IVIP + } 6 \text { months' } \\
\text { work placement; } \\
(n=25) \\
\text { B: standard } \\
\text { support services; }(n=25)\end{array}$ & $\begin{array}{l}N=50 \\
\text { Mean age: } 48.1 \\
\text { Male: } 50 \\
\text { Schizophrenia } \\
\text { spectrum disorder }\end{array}$ & $\begin{array}{l}\text { Vocationally-focused cognitive } \\
\text { behaviour therapy } \\
\text { A: Weekly group and individual } \\
\text { cognitive behaviour therapy } \\
\text { sessions of } 26 \text { weeks. } \\
\text { B: Standard support services. }\end{array}$ & $\begin{array}{l}\text { - Symptom } \\
\text { - Psychological functioning } \\
\text { - Self-esteem } \\
\text { - Vocational functioning } \\
\text { - Employment status }\end{array}$ & $\begin{array}{l}\text { - PANSS: n.s. } \\
\text { BHS: } F(1,48)=5.47 ; p<.05 \\
\text { RSES: } F(1.48)=8.17 ; p<.05 \\
\text { - WBI: } F(1,48)+5.12 ; p<.05 \\
\text { e.s. }=.69 \\
\text { - Weeks of work: } F(1,48)+ \\
\text { 6.03; } p<.02 ; \text { e.s. }=.71\end{array}$ \\
\hline $\begin{array}{l}\text { Fowler } \\
\text { (2009) UK }\end{array}$ & $\begin{array}{l}\text { RCT } \\
2 \text { groups } \\
\text { A: cognitive } \\
\text { behaviour therapy } \\
+ \text { Voc. management } \\
(n=35) \\
\text { B: TAU only }(n=42)\end{array}$ & $\begin{array}{l}N=77 \\
\text { Mean age: } 29 \\
\text { Male: } 55 \\
\text { Female: } 22 \\
\text { Psychosis }\end{array}$ & $\begin{array}{l}\text { Vocationally-focused cognitive } \\
\text { behaviour therapy } \\
\text { A: Social Recovery Cognitive } \\
\text { Behaviour Therapy (SR cognitive } \\
\text { behaviour therapy), } 3 \text { stages } \\
\text { over a 9-month period. } \\
\text { B: Usual care. }\end{array}$ & $\begin{array}{l}\text { - Symptom } \\
\text { - Psychosocial functioning } \\
\text { - Vocational functioning } \\
\text { - Work involvement/ } \\
\text { behaviours }\end{array}$ & $\begin{array}{l}\text { - PANSS: } F(1,44)=4.56 ; \\
\text { p<.05 } \\
\text { QOL: } \text { n.s.; BHS: n.s. } \\
\text { - SOFAS: n.s. } \\
\text { - SC: } F(1,43)=11.4 ; p<.001 \text {; } \\
\text { CEA: } F(1,43)=6.19 ; p<.02\end{array}$ \\
\hline $\begin{array}{l}\text { Temple } \\
\text { (2005) USA }\end{array}$ & $\begin{array}{l}\text { Matching group } \\
\text { pre-test post-test } \\
\text { design } 2 \text { groups } \\
\text { A: cognitive behaviour } \\
\text { therapy }(n=10) \\
\text { B: usual care }(n=9)\end{array}$ & $\begin{array}{l}N=19 \\
\text { Mean age: } 31.5 \\
\text { Male: } 10 \\
\text { Female: } 9 \\
\text { Schizophrenia }\end{array}$ & $\begin{array}{l}\text { General cognitive behaviour } \\
\text { therapy } \\
\text { A: up to } 20 \text { individual general } \\
\text { cognitive behaviour therapy } \\
\text { sessions in } 6 \text { months. } \\
\text { B: Usual care including } \\
\text { medication management and } \\
\text { case management. }\end{array}$ & $\begin{array}{l}\text { - Symptom } \\
\text { - Vocational functioning } \\
\text { - Psychosocial functioning }\end{array}$ & $\begin{array}{l}\text { - Severity of symptom: } \\
d=-.97 ; p<.05 \\
\text { - Occupational impairment: } \\
d=.89, \text { n.s. } \\
\text { - CGI: } d=-1.68, p<.05 \\
\text { GPF: } d=-2.18 ; p<.05 \\
\text { GAS: } d=.79 ; p<.05\end{array}$ \\
\hline $\begin{array}{l}\text { Rose } \\
\text { (2005) } \\
\text { Australia }\end{array}$ & Pre-test Post-test design & $\begin{array}{l}N=27 \\
\text { Mean age: } 34 \\
\text { Male:18 } \\
\text { Schizophrenia }\end{array}$ & $\begin{array}{l}\text { Vocationally-oriented cognitive } \\
\text { behaviour therapy } \\
8 \text { weeks of group cognitive } \\
\text { behaviour therapy program } \\
\text { focused on skills in reframing } \\
\text { negative attributions about work }\end{array}$ & $\begin{array}{l}\text { - Psychosocial functioning } \\
\text { - Work involvement } \\
\text { - Self-esteem }\end{array}$ & $\begin{array}{l}\text { - MCS: } z(13)=-2.062 ; p<.05 \\
\text { LOT z }(12)=-1.931, p<.05 \\
\text { - WIS }(z(12)=-1.931, p<.05 \\
\text { - RSES: } \text { n.s. }\end{array}$ \\
\hline $\begin{array}{l}\text { Lysaker } \\
\text { (2006) USA }\end{array}$ & $\begin{array}{l}\text { RCT } \\
2 \text { groups: } \\
\text { A: IVIP }+6 \text { months' } \\
\text { work placement; }(n=25) \\
\text { B: standard support services; } \\
(n=25)\end{array}$ & $\begin{array}{l}N=50 \\
\text { Mean age: } 48.1 \\
\text { Male: } 50 \\
\text { Schizophrenia spectrum } \\
\text { disorder }\end{array}$ & $\begin{array}{l}\text { Vocationally-oriented cognitive } \\
\text { behaviour therapy } \\
\text { cognitive behaviour therapy } \\
\text { weekly one-hour group sessions } \\
\text { focused on dysfunctional self- } \\
\text { belief and work experience }\end{array}$ & $\begin{array}{l}\text { - Vocational-related } \\
\text { cognition functioning } \\
\text { - Psychosocial functioning }\end{array}$ & $\begin{array}{l}\text { - MAS: } F(1,48)=4.2 ; p<.05 \\
\text { - WCQ acting: } F(1,48,)=4.12 \text {; } \\
p<.05 ; \text { WCQ Considering: } \\
\text { n.s.; WCQ positive } \\
\text { reappraising: } F(1,48)=8.40, \\
p<.01\end{array}$ \\
\hline $\begin{array}{l}\text { Kidd } \\
\text { (2008) Canada }\end{array}$ & $\begin{array}{l}\text { Pre-test post-test } \\
\text { design }\end{array}$ & $\begin{array}{l}N=21 \\
\text { Mean age } 41.3 \\
\text { Mood and anxiety } \\
\text { disorder }\end{array}$ & $\begin{array}{l}\text { Vocationally-focused cognitive } \\
\text { behaviour therapy } \\
5 \text { sessions of vocational-stress } \\
\text { focused cognitive behaviour } \\
\text { therapy group intervention } \\
\text { Pre-test: } 1-2 \text { weeks before } \\
\text { beginning the intervention. } \\
\text { Post-test: the end of the last } \\
\text { group session. }\end{array}$ & $\begin{array}{l}\text { - Job satisfaction } \\
\text { - Vocational functioning } \\
\text { - Expectation for success }\end{array}$ & $\begin{array}{l}\text { - JSS subscales: Job } \\
\text { satisfaction: } \mathrm{n} . \mathrm{s} . ; \text {; pay: } \mathrm{n} . \mathrm{s} \text {.; } \\
\text { advancement and security: } \\
\text { n.s.; supervision: } \text { n.s.; } \\
\text { coworkers: } \text { n.s.; how person } \\
\text { feels on the job; n.s. } \\
\text { - RMMS: } d=-1.14 ; p<.05 \\
\text { - Generalized expectancy for } \\
\text { success: } n \text {.s. }\end{array}$ \\
\hline $\begin{array}{l}\text { Kidorf } \\
\text { (1998) USA }\end{array}$ & Naturalistic evaluation & $\begin{array}{l}N=36 \\
\text { Mean age: } 35.8 \\
\text { Male: } 25 \\
\text { Female: } 11 \\
\text { Opioid dependent outpatient }\end{array}$ & $\begin{array}{l}\text { General cognitive behaviour } \\
\text { therapy+ employment service } \\
\text { Level 1: weekly individual } \\
\text { counselling for } 2 \text { months to } \\
\text { secure employment. } \\
\text { Level 2: Individual and group } \\
\text { counselling. If the participants } \\
\text { fail to keep the employment, } \\
\text { referred to intensive weekly } \\
\text { counselling (i.e. up to } 8 \mathrm{~h} / \text { week) } \\
\text { for } 10 \text { weeks. }\end{array}$ & - Employment status & $\begin{array}{l}\text { Most patients }(78 \%) \text { continued } \\
\text { working throughout the } \\
6 \text {-month. Majority }(56 \%) \\
\text { found paid employment, while } \\
\text { the remainder ( } 44 \% \text { ) started } \\
\text { volunteer work or academic } \\
\text { training. }\end{array}$ \\
\hline $\begin{array}{l}\text { Washington } \\
\text { (1999) USA }\end{array}$ & $\begin{array}{l}\text { Quasi-experimental study } \\
\text { Group1: cognitive } \\
\text { behaviour therapy } \\
\text { Group 2: Experiential } \\
\text { therapy }\end{array}$ & $\begin{array}{l}N=52 \\
\text { Mean age: } 32.67 \\
\text { Female: } 52 \\
\text { Chemically dependent }\end{array}$ & $\begin{array}{l}\text { General cognitive behaviour } \\
\text { therapy } \\
\text { Group interventions } \\
\text { Three weeks interventions }\end{array}$ & $\begin{array}{l}\text { - Self-esteem } \\
\text { - Expectation for job success } \\
\text { - Vocational functioning }\end{array}$ & $\begin{array}{l}\text { - SES general self-efficacy } \\
\text { subscale: } \mathrm{n} . \mathrm{s} . ; \text { SES social self- } \\
\text { efficacy: } p<.05 ; \text { e.s. }=.132 \\
\text { - Ghiselli Self-Description } \\
\text { Inventory subscale: } \\
\text { perception of employability: } \\
F(1,50)=4.34 ; p<.05 \\
\text { - Ghiselli inventory subscale: } \\
\text { Aspiration for employment: } \\
F(1,50)=5.99 ; p<.05 \\
\end{array}$ \\
\hline
\end{tabular}

RCT, randomised controlled trial; n.s., no citation date; e.s., effect size; CORE-OM, Clinical outcomes in routine evaluation - outcome measure; HADS, Hospital Anxiety and Depressions Scale; WSAS, Work and Social Adjustment Scale; PANSS, Positive and Negative Syndrome Scale; WBI, Work Behaviour Inventory; ; CDSS, Calgary Depression Rating Scale for Schizophrenia; CAST, computer-assisted cognitive strategy training; TSSN, training of self-management skills for negative symptoms; BHS, Beck Hopelessness Scale; RSES, Rosenberg self-esteem schedule; QOL, quality of life; SOFAS, Social and Occupational Functioning Assessment Scale; CGI, clinical global impression for improvement; MCS, mental component summary; WIS, Work Involvement Scale; MAS, Metacognitive Assessment Scale; GAS, Global Assessment Scale; RMMS, Relative Mastery Measurement Scale; IVIP, Indianapolis vocational intervention program; $t$, obtained $t$-value; $F$, $F$-value. 
TABLE 2: Proportion of vocational outcome studies and mental illness outcome studies that had significant effects.

\begin{tabular}{|c|c|c|c|c|}
\hline Vocational outcomes & Vocational CBT $(N=17)$ & General CBT $(N=5)$ & $\begin{array}{l}\text { General CBT plus vocationally } \\
\text { relevant }(N=6)\end{array}$ & Overall proportion $(N=28)$ \\
\hline Vocational functioning & 0.75 & 0.50 & - & 0.67 \\
\hline Job satisfaction & 0.00 & - & - & 0.00 \\
\hline Employment status & 1.00 & 1.00 & 1.00 & 1.00 \\
\hline Work adjustment & - & 0.00 & - & 0.00 \\
\hline Work involvement & 1.00 & - & - & 1.00 \\
\hline Expectation of success & 0.00 & 1.00 & - & 0.50 \\
\hline Cognitive functioning & 1.00 & - & 1.00 & 0.60 \\
\hline Total vocational outcomes & 0.53 & 0.60 & 0.67 & 0.57 \\
\hline Mental illness outcomes & $N=30$ & $N=13$ & $N=8$ & $N=51$ \\
\hline Psychological functioning & 1.00 & 0.00 & 0.00 & 0.33 \\
\hline Psychosocial functioning & 0.57 & 1.00 & - & 0.70 \\
\hline Symptom & 0.33 & 0.50 & 0.00 & 0.33 \\
\hline Self-esteem & 0.50 & 0.50 & - & 0.50 \\
\hline
\end{tabular}

$\mathrm{CBT}$, cognitive-behaviour therapy.

those for mental-illness outcomes (0.52). Cognitive-behaviour therapy had significant effects in one out of three results (0.33, see row 12 and the final column). Cognitive-behaviour therapy had a marked effect on psychosocial functioning in seven out of 10 results. Cognitive-behaviour therapy showed statistically significant outcomes in only two out of six cases with respect to symptoms and statistically significant outcomes in two out of four cases with respect to selfesteem. Overall, general cognitive-behaviour therapy was more effective than vocational cognitive-behaviour therapy as far as illness symptoms and outcomes are concerned. In contrast to vocational outcomes, the combination of general cognitive-behaviour therapy plus vocational interventions was the least effective.

\section{Discussion and conclusions}

This systematic review indicated that there is scope for the utilisation of cognitive-behaviour therapy in industrial and organisational settings. Cognitive-behaviour therapy had a positive impact on work-related outcomes for individual with mental illness. Nevertheless, the results of these studies are mixed. Generally speaking, cognitive-behaviour therapy had positive effect on both vocational and mental-illness outcomes.

There was an indication that cognitive-behaviour therapy improved vocational functioning, job satisfaction, vocationalrelated cognitive functioning, employment status and expectation for success amongst people with mental illness. All three approaches to cognitive-behaviour therapy were universally effective in improving employment status, and there was little to justify choosing between vocationally oriented cognitive-behaviour therapy, general cognitivebehaviour therapy or some combination of these two approaches. Amongst three types of cognitive-behaviour therapy, vocationally-oriented cognitive-behaviour therapy is the most effective approach for improving vocational functioning and work involvement, and general cognitivebehaviour therapy is the most effective approach for enhancing expectation for success in people with mental illness. The results of this review indicate that a combination of cognitive-behaviour therapy approaches with vocationally related services is likely to be the first choice by a narrow margin for intervention by organisational and industrial psychologists.

Just over half $(52.1 \%)$ of the findings extracted from the 11 studies indicates that cognitive-behaviour therapy improved psychological functioning, symptoms, self-esteem and psychosocial functioning. The trend of effects favoured general cognitive-behaviour therapy (62\%). This had marginally more positive effects than vocational cognitivebehaviour therapy or a combination of general cognitivebehaviour therapy and vocational approaches combined. The combination of general cognitive-behaviour therapy and vocationally related therapy did not have statistically significant effects on outcomes related to mental illness.

Although this review searched literature that covered a variety of sources and screened studies prior to inclusion, it still has some potential weaknesses. Firstly, as for most reviews, there is always the risk of incomplete retrieval of relevant literature. Moreover, there are some (1) inherent methodological limitations, such as the small sample sizes in the articles and the overall population; (2) the 51 results are mixed, and it is not clear, for instance, why a combination of general cognitive therapy plus vocationally related therapy is not much more effective. The variability of research designs used in these 11 articles also imposes limitations on the findings. There is a need for a randomised trial in industry with substantially larger sample of individuals given the widespread nature of this condition in the population. Longterm follow-up is also required as short-term outcomes are highly variable in employment settings. Moreover, many diverse elements of the approaches to cognitive-behaviour therapy have been categorised neatly in this study, but they overlooked substantive therapeutic differences. Further investigations will be required to determine which therapeutic elements are most relevant for vocational outcomes. Notwithstanding these limitations, the findings seem to indicate that industrial psychologists employing 
cognitive-behaviour therapy as an intervention approach can use this with some confidence in its efficacy. Based on the evidence available, they are likely to achieve improved vocational outcomes, especially employment success, for people with mental illness

\section{Acknowledgements Competing interests}

The authors declare that they have no financial or personal relationship(s) that may have inappropriately influenced them in writing this article.

\section{Authors' contributions}

K.M. (University of Sydney), E.M. (University of Sydney), K.B. (University of Sydney), M.M. (University of Sydney), and J.A. (University of Sydney) contributed equally to the writing of this article.

\section{References}

American Psychiatric Association. (2013). Diagnostic and statistical manual of mental disorders. (5th edn.). Arlington, VA: American Psychiatric Association.

Australian Bureau of Statistics. (1990). Disability and handicap. Catalogue No. 4120.0 Canberra.

Australian Bureau of Statistics. (2008). 2007 National survey of mental health and wellbeing: Summary of results. Catalogue No. 4326.0. Canberra.

Australian Bureau of Statistics. (2013). Disability, ageing \& carers, Australia: Summary of findings, 2012. Catalogue No. 4430.0. Canberra.

Bee, P.E., Bower, P., Gilbody, S., \& Lovell, K. (2010). Improving health and productivity of depressed workers: A pilot randomized controlled trial of telephone cognitive behavioral therapy delivery in workplace settings. General Hospital Psychiatry, 32, 337-340. http://dx.doi.org/10.1016/j.genhosppsych.2010.01.006

Butler, A., Chapman, J., Forman, E., \& Beck, A. (2006). The empirical status of cognitivebehavioral therapy: A review of meta-analyses. Clinical Psychology Review, 26(1), 17-31.

Fowler, D., Hodgekins, J., \& Painter, M., Reilly, T., Crane, C., Macmillian, I. et al. (2009). Cognitive behaviour therapy for improving social recovery in psychosis: A report from the ISREP MRC trial platform study (improving social recovery in early from the ISREP MRC trial platform study (improving social recovery in early psychosis). Psychologicd
S0033291709005467

Garske, G.G., \& Stewart, J.R. (1999). Stigmatic and mythical thinking: Barriers to vocational rehabilitation services for persons with severe mental illness. Journal of Rehabilitation, 65(4), 4-8.

Glynn, L. (2006). EBLIP Critical appraisal checklist. Memorial University of Newfoundland. Retrieved October 02, 2011, from http://www.unisa.edu.au/cahe/resources/cat/ default.asp

Insel, T.R. (2012). Rethinking mental illness: The view from 2022. Article read at the $42 \mathrm{nd}$ National Council on Mentall IIIness and Addictions Conference, Chicago, IL.
Kessler, R.C., Merikangas, K.R., \& Wang, P.S. (2008). The prevalence and correlates of workplace depression in the National Comorbidity Survey Replication. Journal of workplace depression in the National Comorbidity Survey Replication. Journal of
Occupational \& Environmental Medicine, 50, 381-390. http://dx.doi.org/10.1097/ Occupational \& Environm
JOM.0b013e31816ba9b8

Kidd, S.A., Boyd, G.M., Bieling, P., Pike, S., \& Kazarian-Keith, D. (2008). Effect of a vocationally-focused brief cognitive behavioural intervention on employment-related vocationally-focused briefcognitive behavioural intervention on employment-related outcomes for individuals with mood and anxiety disorders. Cognitive Behav
Therapy, 37(4), 247-251. http://dx.doi.org/10.1080/16506070802473189

Kidorf, M. Hollander, J.R., King, V.L., \& Brooner, R.K. (1998). Increasing employment of opioid dependent outpatients: An intensive behavioral intervention. Drug and Alcohol Dependence, 50, 73-80.

Link, B.G., Yang, L.H., Phelan, J.C., \& Collins, P.Y. (2004). Measuring mental illness stigma. Schizophrenia Bulletin, 30(3), 511-541. http://dx.doi.org/10.1093/oxfordjournals. Schizophrenia Bull.a007098
schbula

Lysaker, P.H., Bond, G., Davis, L.W., Bryson, G.J., \& Bell. M.D. (2005). Enhanced cognitivebehavioral therapy for vocational rehabilitation in schizophrenia: Effects on hope and work. Journal or Rehabilitation Research \& Development, 42, 673-682. http:// and work. Journal or Rehabilitation Resear
dx.doi.org/10.1682/JRRD.2004.12.0157

Lysaker, P.H., Davis, L.W., Bryson, G.J., \& Bell, M.D. (2009). Effects of cognitive behavioral therapy on work outcomes in vocational rehabilitation for participants with schizophrenia spectrum disorders. Schizophrenia Research, 107(2), 186-191. http://dx.doi.org/10.1016/j.schres.2008.10.018

Lysaker, P.H., Davis, L.W., \& Beatties, N. (2006). Effects of cognitive behavioral therapy and vocational rehabilitation on metacognition and coping in schizophrenia. Journal of Contemporary Psychotherapy, 36(1), 25-30. http://dx.doi.org/10.1007/s10879of Contempor

Mak, D.C.S., Tsang, H.W.H., \& Cheung, L.C.C. (2006). Job termination among individuals with severe mental illness participating in a supported employment program. with severe mental illness participating in a supported employment
Psychiatry, 69(3), 239-248. http://dx.doi.org/10.1521/psyc.2006.69.3.239

Marwaha, S., \& Johnson, S. (2004). Schizophrenia and employment: A review. Socia Psychiatry and Psychiatric Epidemiology, 39, 337-349. http://dx.doi.org/10.1007/ s00127-004-0762-4

New Freedom Commission on Mental Health. (2003). P.s.N.f.c.o.m.h. Achieving the promise: Transforming the mental health care in America. Rockville, MD: DHSS promise: Transforming the mentc
Publication. No. SMA-03-3832.

Rachman, S. (1997). The evolution of cognitive behaviour therapy. In D. Clark, C.G. Fairburn \& M.G. Gelder, (Eds.), Science and practice of cognitive behaviour therapy (pp. 1-26). Oxford: Oxford University Press.

Rose, V., \& Perz, J. (2005). Is cognitive behaviour therapy useful in vocational rehabilitation for people with a psychiatric disability? Psychiatric Rehabilitation Journal, 29(1), 56-58. http://dx.doi.org/10.2975/29.2005.56.58

Temple, S., \& Ho, B.C. (2005). Cognitive therapy for persistent psychosis in schizophrenia: A case-controlled clinical trial. Schizophrenia Research, 74, 195-199.

Tsang, H., Lam, P., Ng, B. \& Leung, O. (2000). Predictors of employment outcome for people with psychiatric disabilities: A review of the literature since the mid ' 80 s. Journal of Rehabilitation, 66, 19-31.

Vauth, R., Corrigan, P.W., Clauss, M., Dietl, M., Dreher-Rudolph, M., Stieglitz, R-D. et al (2005). Cognitive strategies verse self-management skills as adjunct to vocational rehabilitation. Schizophrenia Bulletin, 31, 55-66. http://dx.doi.org/10.1093/schbul/ sbi013

Washington, O. (1999). Effects of cognitive and experiential group therapy on selfefficacy and perceptions of employability of chemically dependent women. Mental Health Nursing, 20, 181-198. http://dx.doi.org/10.1080/016128499248600

World Health Organization. (2001). Mental health: New understanding, new hope. Geneva: WHO. Retrieved March 05, 2014, from http://www.who.int/whr/2001/en/

World Health Organization. (2007). Mental health: Strengthening mental health promotion (fact sheet no. 220). Geneva: WHO. Retrieved March 2014, from http:// www.who.int/mediacentre/factsheets/fs220/en/ 